\title{
Hamiltonian chromatic number of trees
}

\author{
Devsi Bantva \\ Department of Mathematics \\ Lukhdhirji Engineering College, Morvi - 363 642, Gujarat (INDIA) \\ E-mail : devsi.bantva@gmail.com \\ Samir Vaidya \\ Department of Mathematics \\ Saurashtra University, Rajkot - 360 005, Gujarat (INDIA) \\ E-mail : samirkvaidya@yahoo.co.in
}

\begin{abstract}
Let $G$ be a simple finite connected graph of order $n$. The detour distance between two distinct vertices $u$ and $v$ denoted by $D(u, v)$ is the length of a longest $u v$-path in $G$. A hamiltonian coloring $h$ of a graph $G$ of order $n$ is a mapping $h: V(G) \rightarrow\{0,1,2, \ldots\}$ such that $D(u, v)+|h(u)-h(v)| \geq n-1$, for every two distinct vertices $u$ and $v$ of $G$. The span of $h$, denoted by $\operatorname{span}(h)$, is $\max \{|h(u)-h(v)|: u, v \in V(G)\}$. The hamiltonian chromatic number of $G$ is defined as hc $(G):=\min \{\operatorname{span}(h)\}$ with minimum taken over all hamiltonian coloring $h$ of $G$. In this paper, we give an improved lower bound for the hamiltonian chromatic number of trees and give a necessary and sufficient condition to achieve the improved lower bound. Using this result, we determine the hamiltonian chromatic number of two families of trees.
\end{abstract}

\section{Introduction}

Let $G$ be a simple finite connected graph with vertex set $V(G)$ and edge set $E(G)$. The order of a graph $G$ written as $|G|$ is the number of vertices in $G$. For a vertex $v \in V(G)$, the neighborhood of $v$ denoted by $N(v)$ is the set of vertices adjacent to $v$. The distance $d(u, v)$ between two vertices $u$ and $v$ is the length of a shortest path joining $u$ and $v$. The detour distance between two vertices $u$ and $v$ denoted by $D(u, v)$ is the length of a longest path joining $u$ and $v$ (refer [6] for more details on it). The diameter of a graph $G$ denoted by $\operatorname{diam}(G)$ or simply $d$ is $\max \{d(u, v): u, v \in V(G)\}$. The eccentricity $\epsilon(v)$ of a vertex $v \in V(G)$ is the distance from $v$ to a vertex farthest from $v$. The center $C(G)$ of graph $G$ is the subgraph of $G$ induced by the vertex/vartices of $G$ whose eccentricity is minimum. Moreover, for standard graph theoretic terminology and notation we follow [10.

A hamiltonian coloring $h$ of a graph $G$, introduced by Chartrand et al. in [4], is a mapping $h: V(G) \rightarrow\{0,1,2, \ldots\}$ such that for every pair of distinct vertices $u, v$ of $G$,

$$
D(u, v)+|h(u)-h(v)| \geq n-1 .
$$

The span of $h$, denoted by $\operatorname{span}(h)$, is defined as $\max \{|h(u)-h(v)|: u, v \in V(G)\}$. The hamiltonian chromatic number $\mathrm{hc}(G)$ of $G$ is

$$
\operatorname{hc}(G):=\min \{\operatorname{span}(h)\}
$$

with minimum taken over all hamiltonian coloring $h$ of $G$. A hamiltonian coloring $h$ of $G$ is called optimal if $\operatorname{span}(h)=\mathrm{hc}(G)$. 
It is clear from definition that if $G$ contains a hamiltonian $u v$-path between two distinct vertices $u$ and $v$ then the same color can be assigned to both $u$ and $v$. Hence, a graph $G$ is hamiltonian-connected if and only if $G$ can be hamiltonian colored by a single color. In [4, Chartrand et al. proved that for any two integers $j$ and $n$ with $2 \leq j \leq(n+1) / 2$ and $n \geq 6$, there is a hamiltonian graph of order $n$ with hamiltonian chromatic number $n-j$. Thus the hamiltonian chromatic number of a connected graph $G$ measures how close $G$ is to being hamiltonian-connected. Without loss of generality we allow 0 as a color in the definition of the hamiltonian coloring, then the span of any hamiltonian coloring $h$ is the maximum integer used for coloring while in [4, 5, 9] only positive integers are used as colors. Therefore, the hamiltonian chromatic number defined in this article is one less than that defined in [4, 5, 9] and hence we will make necessary adjustment when we present the results of [4, 5, 9] in this article.

The hamiltonian chromatic number was introduced by Chartrand et al. in [4] as a variation of radio antipodal coloring of graphs but it is less explored compare to it. A very few results presented by researchers for hamiltonian chromatic number of graphs. The hamiltonian chromatic number of some well-known graph families is determined by Chartrand et al. in [4] which are as follows: $\mathrm{hc}\left(K_{n}\right)=0$ for $n \geq 1, \mathrm{hc}\left(C_{n}\right)=n-3$ for $n \geq 3$, hc $\left(K_{1, n-1}\right)=(n-2)^{2}$ for $n \geq 3$, $\mathrm{hc}\left(K_{r, r}\right)=r-1$ for $r \geq 1$, hc $\left(K_{r, s}\right)=(s-1)^{2}-(r-1)^{2}-1$ for $2 \leq r<s$ and gave an upper bound for $\mathrm{hc}\left(P_{n}\right)$. However, it is noted that $h c\left(P_{n}\right)$ is same as radio antipodal number $a c\left(P_{n}\right)$ which is determined in [7. They proved that if $T$ is a spanning tree of a connected graph $G$ then $\mathrm{hc}(G) \leq \mathrm{hc}(T)$ and for any tree $T$ of order $n \geq 2, \mathrm{hc}(T) \leq(n-2)^{2}$. They also proved that for any two integers $j$ and $n$ with $2 \leq j \leq(n+1) / 2$ and $n \geq 6$, there is a hamiltonian graph of order $n$ with hamiltonian chromatic number $n-j$. In [5], the same group of authors gave a lower bound for circumference of $G$ (the circumference of a graph $G$ is the length of a longest cycle in $G$ ) is given in terms of the number of vertices that receive colors between two specified colors in a hamiltonian coloring of $G$. The authors also proved that for a connected graph $G$ of order $n \geq 3$, if $(n+2) / 2$ vertices receive the same color in a hamiltonian coloring then $G$ is hamiltonian. In [9], Shen et al. determined the hamiltonian chromatic number of graph $G$ with $\max \{D(u, v): u, v \in V(G), u \neq v\} \leq n / 2$ and illustrated the result with a special class of caterpillars and double stars.

This paper is organized as follows. In section 2, we define all necessary terms, notations and terminologies for present work. In section 3 , we give improved lower bound for the hamiltonian chromatic number of trees and present a necessary and sufficient condition to achieve the improved lower bound. We determine the hamiltonian chromatic number of two families of trees in section 4. In this section, we show that for a special type of broom trees (see section 4.2 for definition and detail on it) the lower bound given in present work is better than one given in [3, Theorem 4]. We explain the strength of our results in concluding remarks section.

\section{Preliminaries}

A tree $T$ is a connected graph that contains no cycle. In [8] and later used in [2], the weight of $T$ from $v \in V(T)$ is defined as $w_{T}(v)=\sum_{u \in V(T)} d(u, v)$ and the weight of $T$ as $w(T)=$ $\min \left\{w_{T}(v): v \in V(T)\right\}$. A vertex $v \in V(T)$ is a weight center of $T$ if $w_{T}(v)=w(T)$. Denote the set of wight centers of $T$ by $W(T)$. It was proved in [8] that every tree $T$ has either one or two weight centers, and $T$ has two weight centers, say $W(T)=\left\{w, w^{\prime}\right\}$, if and only if $w$ and $w^{\prime}$ are adjacent and $T-\left\{w w^{\prime}\right\}$ consists of two equal-sized components. We set $W(T)=\{w\}$ as a root if $T$ has only one weight center $w$ and $W(T)=\left\{w, w^{\prime}\right\}$ as a root if $T$ has two adjacent 
weight centers $w$ and $w^{\prime}$. In either case, if a vertex $u$ is on the path joining weight center and a vertex $v \in V(T)$ then $u$ is called ancestor of $v$ and $v$ is called descendent of $u$. If $u$ is ancestor of $v$ which is adjacent to $v$ then $u$ is called parent of $v$ and $v$ is called a child of $u$. The subtree induced by a child $u$ of a weight center and all descendent of $u$ is called branch at $u$. Two branches are called different if they are at two vertices adjacent to the same weight center, and opposite if they are at two vertices adjacent to different weight centers. Note that the concept of opposite branches occurs only when $T$ has two weight centers.

Define

$$
\mathcal{L}(u):=\min \{D(u, w): w \in W(T)\}, u \in V(T)
$$

to indicate the detour level of $u$ in $T$. Define the total detour level of $T$ as

$$
\mathcal{L}_{W}(T):=\sum_{u \in V(T)} \mathcal{L}(u)
$$

For any $u, v \in V(T)$, define

$$
\phi(u, v):=\max \{\mathcal{L}(t): t \text { is a common ancestor of } u \text { and } v\},
$$

$$
\delta(u, v):= \begin{cases}1, & \text { If } W(T)=\left\{w, w^{\prime}\right\} \text { and } u v \text {-path contains the edge } w w^{\prime} \\ 0, & \text { otherwise }\end{cases}
$$

Lemma 2.1. Let $T$ be a tree with diameter $d \geq 2$. Then for any $u, v \in V(T)$, the following hold:

(a) $\phi(u, v) \geq 0$;

(b) $\phi(u, v)=0$ if and only if $u$ and $v$ are in different or opposite branches;

(c) $\delta(u, v)=1$ if and only if $T$ has two weight centers and $u$ and $v$ are in opposite branches.

(d) the detour distance $D(u, v)$ in $T$ between $u$ and $v$ can be expressed as

$$
D(u, v)=\mathcal{L}(u)+\mathcal{L}(v)-2 \phi(u, v)+\delta(u, v)
$$

Note that the detour distance $D(u, v)$ is same as the ordinary distance $d(u, v)$ between any two vertices $u$ and $v$ in a tree $T$ but we continue to use this terminology to remain consistent with concept of hamiltonian coloring in general.

Define

$$
\zeta(T):= \begin{cases}0, & \text { If } W(T)=\{w\}, \\ 1, & \text { If } W(T)=\left\{w, w^{\prime}\right\}\end{cases}
$$

and

$$
\zeta^{\prime}(T):=1-\zeta(T)
$$




\section{A lower bound for hc(T)}

In this section, we continue to use terms, notations and terminologies defined in previous section.

A hamiltonian coloring $h$ of a tree $T$ is injective if $T$ has at least one vertex of degree 3, that is, $T$ is not a path as in this case no two vertices of trees contain hamiltonian path. So we assume all trees with at least one vertex of degree 3 through out this discussion unless otherwise specified. Observe that a hamiltonian coloring $h$ on $V(T)$, induces an ordering of $V(T)$, which is a line-up of the vertices with increasing images. We denote this ordering by $V(G)=\left\{x_{0}, x_{1}, x_{2}, \ldots, x_{n-1}\right\}$ with

$$
0=h\left(x_{0}\right)<h\left(x_{1}\right)<\ldots<h\left(x_{n-1}\right)=\operatorname{span}(h) .
$$

The next result gives a lower bound for the hamiltonian chromatic number of trees.

Theorem 3.1. Let $T$ be a tree of order $n \geq 4$ and $\Delta(T) \geq 3$. Then

$$
\operatorname{hc}(T) \geq(n-1)(n-1-\zeta(T))+\zeta^{\prime}(T)-2 \mathcal{L}_{W}(T) .
$$

The proof is similar to that of [3, Theorem 4] except only one change which is $\mathcal{L}(T)$ is replaced by $\mathcal{L}_{W}(T)$ but it should be noted that technically this lower bound is more useful than one given in [3, Theorem 4]. We support this fact by giving an example in section 4 .

Theorem 3.2. Let $T$ be a tree of order $n \geq 4$ and $\Delta(T) \geq 3$. Then

$$
\mathrm{hc}(T) \geq(n-1)(n-1-\zeta(T))+\zeta^{\prime}(T)-2 \mathcal{L}_{W}(T)
$$

holds if and only if there exist an ordering $\left\{x_{0}, x_{1}, \ldots, x_{n-1}\right\}$ of the vertices of $T$, with $\mathcal{L}\left(x_{0}\right)=$ 0 and $\mathcal{L}\left(x_{n-1}\right)=1$ when $W(T)=\{w\}$ and $\mathcal{L}\left(x_{0}\right)=\mathcal{L}\left(x_{n-1}\right)=0$ when $W(T)=\left\{w, w^{\prime}\right\}$, such that for all $0 \leq i<j \leq n-1$,

$$
D\left(x_{i}, x_{j}\right) \geq \sum_{t=i}^{j-1}\left(\mathcal{L}\left(x_{t}\right)+\mathcal{L}\left(x_{t+1}\right)\right)-(j-i)(n-1-\zeta(T))+(n-1) .
$$

Moreover, under this condition the mapping $h$ defined by

$$
\begin{gathered}
h\left(x_{0}\right)=0 \\
h\left(x_{i+1}\right)=h\left(x_{i}\right)+n-1-\zeta(T)-\mathcal{L}\left(x_{i}\right)-\mathcal{L}\left(x_{i+1}\right) .
\end{gathered}
$$

Proof. Necessity: Suppose that (4) holds. Let $h$ be an optimal hamiltonian coloring of $T$ then $h$ induces an ordering of vertices of $T$, say $0=h\left(x_{0}\right)<h\left(x_{1}\right)<\ldots<h\left(x_{n-1}\right)$. The span of $h$ is $\operatorname{span}(h)=\operatorname{hc}(T)=(n-1)(n-1-\zeta(T))+\zeta^{\prime}(T)-2 \mathcal{L}_{W}(T)$. Note that this is possible if equality holds in (1) together with (a) $\mathcal{L}\left(x_{0}\right)=0, \mathcal{L}\left(x_{n-1}\right)=1$ and $\phi\left(x_{i}, x_{i+1}\right)=\delta\left(x_{i}, x_{i+1}\right)$ $=0$ when $T$ has only one weight center, (b) $\mathcal{L}\left(x_{0}\right)=\mathcal{L}\left(x_{n-1}\right)=0$ and $\phi\left(x_{i}, x_{i+1}\right)=0$ and $\delta\left(x_{i}, x_{i+1}\right)=1$ when $T$ has two adjacent weight centers. Note that this turn the definition of hamiltonian coloring $h\left(x_{i+1}\right)-h\left(x_{i}\right)=n-1-D\left(x_{i}, x_{i+1}\right)$ to $h\left(x_{0}\right)=0$ and $h\left(x_{i+1}\right)=$ $h\left(x_{i}\right)+n-1-\zeta(T)-\mathcal{L}\left(x_{i}\right)-\mathcal{L}\left(x_{i+1}\right)$ for $0 \leq i \leq n-2$. Moreover, for any two vertices $x_{i}$ and $x_{j}$ (without loss of generality, assume $j>i$ ), summing the latter equality for index $i$ to $j$, we have

$$
h\left(x_{j}\right)-h\left(x_{i}\right)=\sum_{t=i}^{j-1}\left[n-1-\zeta(T)-\mathcal{L}\left(x_{t}\right)-\mathcal{L}\left(x_{t+1}\right)\right] .
$$


Now $h$ is a hamiltonian coloring so that $h\left(x_{j}\right)-h\left(x_{i}\right) \geq n-1-D\left(x_{i}, x_{j}\right)$ which turn the above equation in the following form.

$$
D\left(x_{i}, x_{j}\right) \geq \sum_{t=i}^{j-1}\left[\mathcal{L}\left(x_{t}\right)+\mathcal{L}\left(x_{t+1}\right)\right]-(j-i)(n-1-\zeta(T))+(n-1) .
$$

Sufficiency: Suppose that an ordering $\left\{x_{0}, x_{1}, \ldots, x_{n-1}\right\}$ of vertices of $T$ satisfies (5), and $h$ is defined by (6) and (7) together with $\mathcal{L}\left(x_{0}\right)=0, \mathcal{L}\left(x_{n-1}\right)=1$ when $W(T)=\{w\}$ and $\mathcal{L}\left(x_{0}\right)=$ $\mathcal{L}\left(x_{n-1}\right)=0$ when $W(T)=\left\{w, w^{\prime}\right\}$. Note that it is enough to prove that $h$ is a hamiltonian coloring with span equal to the right-hand side of (4). Let $x_{i}$ and $x_{j}(j>i)$ be two arbitrary vertices then by (7), we have

$$
h\left(x_{j}\right)-h\left(x_{i}\right)=(j-i)(n-1-\zeta(T))-\sum_{t=i}^{j-1}\left[\mathcal{L}\left(x_{t}\right)+\mathcal{L}\left(x_{t+1}\right)\right]
$$

Now an ordering satisfies (5) which turn the above equation in the following form which shows that $h$ is a hamiltonian coloring.

$$
h\left(x_{j}\right)-h\left(x_{i}\right) \geq n-1-D\left(x_{i}, x_{j}\right) .
$$

The span of $h$ is given by

$$
\begin{aligned}
\operatorname{span}(h) & =h\left(x_{n-1}\right)-h\left(x_{0}\right) \\
& =\sum_{i=0}^{n-2}\left(h\left(x_{i+1}\right)-h\left(x_{i}\right)\right) \\
& =(n-1)(n-1-\zeta(T))-\sum_{i=0}^{n-2}\left(\mathcal{L}\left(x_{i}\right)+\mathcal{L}\left(x_{i+1}\right)\right) \\
& =(n-1)(n-1-\zeta(T))-2 \mathcal{L}_{W}(T)+\mathcal{L}\left(x_{0}\right)+\mathcal{L}\left(x_{n-1}\right) \\
& =(n-1)(n-1-\zeta(T))+\zeta^{\prime}(T)-2 \mathcal{L}_{W}(T)
\end{aligned}
$$

which complete the proof.

Note that the following results gives sufficient conditions with optimal hamiltonian coloring for the equality in (3). Note that these results are identical with [3, Theorem 5] and [3, Corollary 1] when $W(T)=C(T)$ for a tree $T$. But it should be note that these results are more efficient than [3, Theorem 5] and [3, Corollary 1] (see the case of broom trees in section 4.2).

Theorem 3.3. Let $T$ be a tree of order $n \geq 4$ and $\Delta(T) \geq 3$. Then

$$
\operatorname{hc}(T)=(n-1)(n-1-\zeta(T))+\zeta^{\prime}(T)-2 \mathcal{L}_{W}(T)
$$

holds if there exist an ordering $\left\{x_{0}, x_{1}, \ldots, x_{n-1}\right\}$ of the vertices of $T$ such that for all $0 \leq i \leq$ $n-2$,

(a) $\mathcal{L}\left(x_{0}\right)+\mathcal{L}\left(x_{n-1}\right)=1$ when $W(T)=\{w\}$ and $\mathcal{L}\left(x_{0}\right)+\mathcal{L}\left(x_{n-1}\right)=0$ when $W(T)=\left\{w, w^{\prime}\right\}$,

(b) $x_{i}$ and $x_{i+1}$ are in different branches when $W(T)=\{w\}$ and in opposite branches when $W(T)=\left\{w, w^{\prime}\right\}$, 
(c) $D\left(x_{i}, x_{i+1}\right) \leq n / 2$.

Moreover, under these conditions the mapping $h$ defined by (6) and (7) is an optimal hamiltonian coloring of $T$.

Proof. Suppose there exist an ordering $\left\{x_{0}, x_{1}, \ldots, x_{n-1}\right\}$ of vertices of $T$ such that (a), (b) and (c) holds and $h$ is defined by (6) and (7). By Theorem 3.2, it is enough to prove that an ordering $\left\{x_{0}, x_{1}, \ldots, x_{n-1}\right\}$ satisfies (5). Let $x_{i}$ and $x_{j}$ be two arbitrary vertices, where $0 \leq i<j \leq n-1$. Without loss of generality, we assume that $j-i \geq 2$ and for simplicity let the right-hand side of (5) is $x_{i, j}$. Then, we obtain

$$
\begin{aligned}
x_{i, j} & =\sum_{t=i}^{j-1}\left[\mathcal{L}\left(x_{t}\right)+\mathcal{L}\left(x_{t+1}\right)\right]-(j-i)(n-1-\zeta(T))+(n-1) \\
& \leq(j-i)(n / 2-\zeta(T))-(j-i)(n-1-\zeta(T))+(n-1) \\
& =(j-i)(1-n / 2)+(n-1) \\
& \leq 2(1-n / 2)+(n-1) \\
& =1 \leq D\left(x_{i}, x_{j}\right)
\end{aligned}
$$

which completes the proof.

For a tree $T$ of order $n$, if $\max \{d(u, v): u, v \in V(T), u \neq v\} \leq n / 2$ then such a tree $T$ is called a tree with maximum distance bound $n / 2$ or $D B(n / 2)$ tree.

Corollary 3.4. Let $T$ be a $D B(n / 2)$ tree of order $n \geq 4$ and $\Delta(T) \geq 3$. Then

$$
\operatorname{hc}(T)=(n-1)(n-1-\zeta(T))+\zeta^{\prime}(T)-2 \mathcal{L}_{W}(T)
$$

holds if and only if there exist an ordering $\left\{x_{0}, x_{1}, \ldots, x_{n-1}\right\}$ of the vertices of $T$ such that for all $0 \leq i \leq n-2$,

(a) $\mathcal{L}\left(x_{0}\right)+\mathcal{L}\left(x_{n-1}\right)=1$ when $W(T)=\{w\}$ and $\mathcal{L}\left(x_{0}\right)+\mathcal{L}\left(x_{n-1}\right)=0$ when $W(T)=\left\{w, w^{\prime}\right\}$,

(b) $x_{i}$ and $x_{i+1}$ are in different branches when $W(T)=\{w\}$ and in opposite branches when $W(T)=\left\{w, w^{\prime}\right\}$,

Moreover, under these conditions the mapping h defined by (6) and (7) is an optimal hamiltonian coloring of $T$.

\section{Theorem 1 vs [3, Theorem 4]}

In this section, we determine the hamiltonian chromatic number of two families of trees namely $A_{d}$ and $B_{d}$, where $d=2 k, 2 k+1$ and $k \geq 1$ be any integer. We show that in case of $W(T)=C(T)$, the lower bound for hc(T) given in Theorem 3 and [3, Theorem 4] are identical but in case of $W(T) \neq C(T)$ then the lower bound for hc $(T)$ given in Theorem 3 is better than [3, Theorem 4]. Note that in case of $A_{d}, W\left(A_{d}\right)=C\left(A_{d}\right)$ but in case of $B_{d}, W\left(B_{d}\right) \neq C\left(B_{d}\right)$, where $d=2 k, 2 k+1$. 


\subsection{Special Trees $A_{2 k}$ and $A_{2 k+1}$}

In [1, a special class of trees, namely $A_{2 k}$ and $A_{2 k+1}$, were introduced. The trees $A_{2 k}$ with diameter $2 k-1$ is defined as follows. (a) $A_{2}$ is an edge $K_{2}$, (b) $A_{2 k}(k \geq 2)$ is obtained from $A_{2 k-2}$ in the following manner: Add three pendant vertices (two vertical and one horizontal) to the left most and the right most pendant vertices of $A_{2 k-2}$; next, add a pendant vertex (vertically) to every other pendant vertex of $A_{2 k-2}$. In a manner analogous to the above, define the class of trees $A_{2 k+1}(k \geq 1)$ with diameter $2 k$ starting with $A_{3}$ which is $K_{1,4}$. The trees $A_{2 k}$ and $A_{2 k+1}$ has $2 k^{2}$ and $2 k(k+1)+1$ vertices respectively. Note that $\left|W\left(A_{2 k}\right)\right|=2$ and $\left|W\left(A_{2 k+1}\right)\right|=1$. Denote $W\left(A_{2 k}\right)=\left\{w, w^{\prime}\right\}$ and $W\left(A_{2 k+1}\right)=\{w\}$. Moreover, it is clear from definition that $A_{2 k}$ and $A_{2 k+1}$ are $D B(n / 2)$ trees.

Theorem 4.1. Let $k \geq 1$ be any integer. Then

$$
\operatorname{hc}\left(A_{d}\right):= \begin{cases}\frac{2}{3}(k-1)\left(6 k^{3}+2 k^{2}-4 k-3\right), & \text { If } d=2 k, \\ \frac{1}{3} k(k+1)\left(3 k^{2}+k-1\right)+1, & \text { If } d=2 k+1 .\end{cases}
$$

Proof. The order $n$ and the total detour level $\mathcal{L}_{W}\left(A_{d}\right)$ of $A_{d}(d=2 k, 2 k+1)$ are given by

$$
\begin{gathered}
n:= \begin{cases}2 k^{2}, & \text { If } d=2 k, \\
2 k(k+1)+1, & \text { If } d=2 k+1 .\end{cases} \\
\mathcal{L}_{W}\left(A_{d}\right):= \begin{cases}\frac{1}{3} k(k-1)(4 k+1), & \text { If } d=2 k, \\
\frac{2}{3} k(k+1)(2 k+1), & \text { If } d=2 k+1 .\end{cases}
\end{gathered}
$$

Substitute (11) and (12) into (3) we obtain the right-hand side of 10 is a lower bound for hc $\left(A_{d}\right)$ $(d=2 k, 2 k+1)$. Now we prove that this lower bound is tight and for this purpose it suffices to give a hamiltonian coloring whose span equal to the right-hand side of 10$)$. Since $A_{d}(d=$ $2 k, 2 k+1)$ are $D B(n / 2)$ tree, by Corollary 3.4 it is enough to give an ordering $\left\{x_{0}, x_{1}, \ldots, x_{n-1}\right\}$ of $V\left(A_{d}\right)(d=2 k, 2 k+1)$ satisfying conditions (a) and (b) of Corollary 3.4 and the hamiltonian coloring $h$ defined by (6)-(7) is an optimal hamiltonian coloring. For this we consider the following two cases for $A_{d}$.

Case-1: $d=2 k$. In this case, note that $\left|W\left(A_{2 k}\right)\right|=2$ and $A_{2 k}$ has six branches. Denote the branches of $A_{2 k}$ attached to $w$ by $T_{2}, T_{4}, T_{6}$ and $w^{\prime}$ by $T_{1}, T_{3}, T_{5}$ such that $\left|T_{2}\right|>\left|T_{4}\right|=\left|T_{6}\right|$ and $\left|T_{1}\right|>\left|T_{3}\right|=\left|T_{5}\right|$. Define an ordering $\left\{x_{0}, x_{1}, \ldots, x_{n-1}\right\}$ of $V\left(A_{2 k}\right)$ as follows: Let $x_{0}=w$ and $x_{n-1}=w^{\prime}$. For $1 \leq i \leq n-4 k-1$, let

$$
x_{i}:= \begin{cases}v \in V\left(T_{1}\right), & \text { If } i \text { is odd, } \\ v \in V\left(T_{2}\right), & \text { If } i \text { is even. }\end{cases}
$$

For $n-4 k \leq i \leq n-2 k-1$, let

$$
x_{i}:= \begin{cases}v \in V\left(T_{3}\right), & \text { If } i \text { is odd, } \\ v \in V\left(T_{4}\right), & \text { If } i \text { is even. }\end{cases}
$$

For $n-2 k \leq i \leq n-2$, let

$$
x_{i}:= \begin{cases}v \in V\left(T_{5}\right), & \text { If } i \text { is odd, } \\ v \in V\left(T_{6}\right), & \text { If } i \text { is even. }\end{cases}
$$


In above ordering $v \in V\left(T_{i}\right)$, where $i=1,2, \ldots, 6$, we mean any $v$ from $V\left(T_{i}\right)$ without repetition. Note that $\mathcal{L}\left(x_{0}\right)+\mathcal{L}\left(x_{n-1}\right)=0$ and $x_{i}, x_{i+1}(0 \leq i \leq n-1)$ are in opposite branches of $A_{2 k}$.

Case-2: $d=2 k+1$. In this case, note that $\left|W\left(A_{2 k+1}\right)\right|=1$ and $A_{2 k+1}$ has four branches. Denote the branches of $A_{2 k+1}$ attached to $w$ by $T_{1}, T_{2}, T_{3}, T_{4}$ such that $\left|T_{1}\right|=\left|T_{2}\right|>\left|T_{3}\right|=\left|T_{4}\right|$. Define an ordering $\left\{x_{0}, x_{1}, \ldots, x_{n-1}\right\}$ of $V\left(A_{2 k+1}\right)$ as follows: Let $x_{0}=w$ and $x_{n-1}=v \in V\left(T_{4}\right)$ such that $v$ is adjacent to $w$. For $1 \leq i \leq n-2 k-1$, let

$$
x_{i}:= \begin{cases}v \in V\left(T_{1}\right), & \text { If } i \text { is odd, } \\ v \in V\left(T_{2}\right), & \text { If } i \text { is even. }\end{cases}
$$

For $n-2 k \leq i \leq n-2$, let

$$
x_{i}:= \begin{cases}v \in V\left(T_{3}\right), & \text { If } i \text { is odd, } \\ v \in V\left(T_{4}\right), & \text { If } i \text { is even. }\end{cases}
$$

Again in above ordering $v \in V\left(T_{i}\right)$, where $i=1,2,3,4$, we mean any $v$ from $V\left(T_{i}\right)$ without repetition. Note that $\mathcal{L}\left(x_{0}\right)+\mathcal{L}\left(x_{n-1}\right)=1$ and $x_{i}, x_{i+1}(0 \leq i \leq n-1)$ are in different branches of $A_{2 k+1}$.

Remark 4.2. Note that in case of $A_{d}(d=2 k, 2 k+1), W\left(A_{d}\right)=C\left(A_{d}\right)$ and hence both the lower bounds given in Theorem 3.1 and [3, Theorem 4] are identical. Moreover, $\Delta\left(A_{2}\right)=1$ but it is easy to verify that $\mathrm{hc}\left(A_{2}\right)=0$ and the case of $A_{2}$ is also consistent with the formula in (10).

Example 4.3. An optimal hamiltonian coloring of $A_{2}, A_{4}, A_{6}$ and $A_{3}, A_{5}, A_{7}$ is shown in Fig 1 .

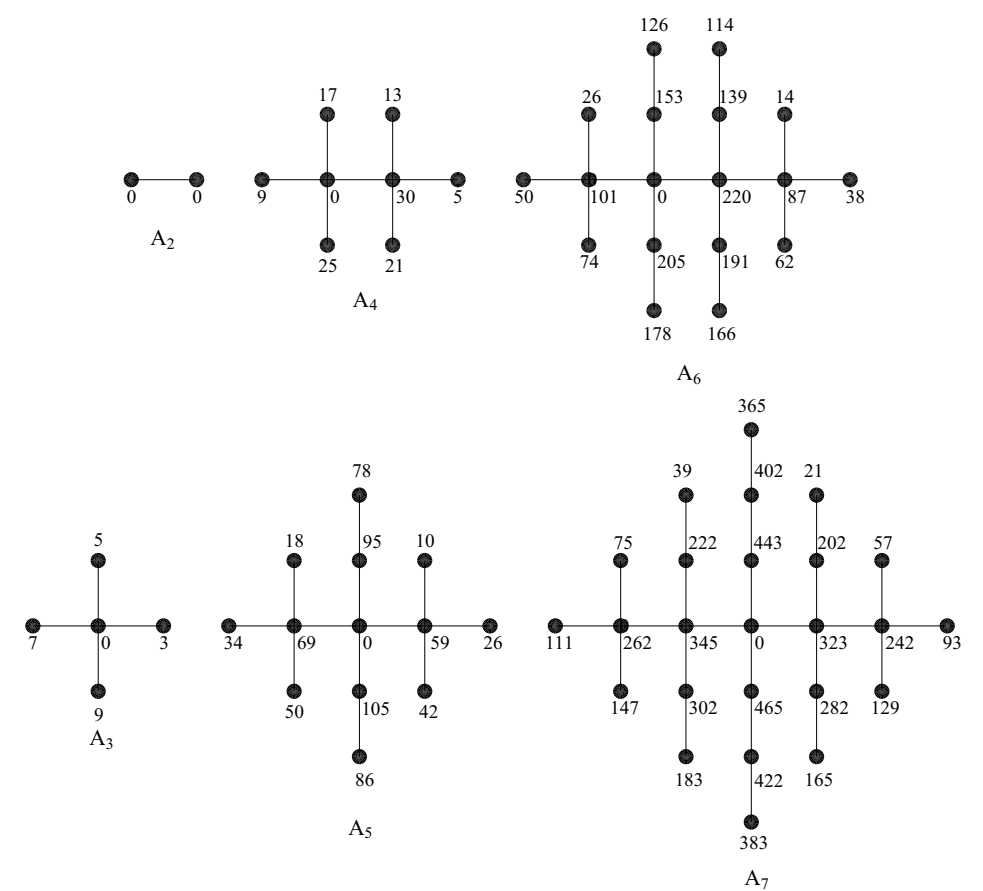

Figure 1: An optimal hamiltonian coloring of $A_{2}, A_{4}, A_{6}$ and $A_{3}, A_{5}, A_{7}$. 


\subsection{Broom Trees $B_{2 k}$ and $B_{2 k+1}$}

The broom tree $B_{n, d}$ consists of a path $P_{d}$, together with $(n-d)$ end vertices all adjacent to the same end vertex of $P_{d}$. Let $k \geq 1$ be any integer. Define the broom trees $B_{2 k}$ and $B_{2 k+1}$ are broom tree $B_{k(2 k+1), 2 k}$ and $B_{(k+1)(2 k+1), 2 k+1}$, respectively. It is clear that $\left|W\left(B_{2 k}\right)\right|=$ $\left|W\left(B_{2 k+1}\right)\right|=1$. Denote $W\left(B_{2 k}\right)=W\left(B_{2 k+1}\right)=\{w\}$. Moreover, it is clear from definition that $B_{2 k}$ and $B_{2 k+1}$ are $D B(n / 2)$ trees.

Theorem 4.4. Let $k \geq 1$ be any integer. Then

$$
h c\left(B_{d}\right):= \begin{cases}2 k\left(2 k^{3}+2 k^{2}-\frac{11}{2} k+1\right)+2, & \text { If } d=2 k, \\ (2 k+1)\left(2 k^{3}+5 k^{2}-2 k-1\right)+2, & \text { If } d=2 k+1 .\end{cases}
$$

Proof. The order $n$ and the total detour level $\mathcal{L}_{W}\left(B_{d}\right)$ of $B_{d}(d=2 k, 2 k+1)$ are given by

$$
\begin{gathered}
n:= \begin{cases}k(2 k+1), & \text { If } d=2 k, \\
(k+1)(2 k+1), & \text { If } d=2 k+1 .\end{cases} \\
\mathcal{L}_{W}\left(B_{d}\right):= \begin{cases}2 k(2 k-1), & \text { If } d=2 k, \\
2 k(2 k+1), & \text { If } d=2 k+1 .\end{cases}
\end{gathered}
$$

Substitute (14) and (15) into (3) we obtain the right-hand side of (13) is a lower bound for hc $\left(B_{d}\right) \quad(d=2 k, 2 k+1)$. Now we prove that this lower bound is tight and for this purpose it is suffices to give a hamiltonian coloring whose span equal to the right-hand side of (13). Since $B_{d}(d=2 k, 2 k+1)$ are $D B(n / 2)$ tree, by Corollary 3.4 it is enough to give an ordering $\left\{x_{0}, x_{1}, \ldots, x_{n-1}\right\}$ of $V\left(B_{d}\right)(d=2 k, 2 k+1)$ satisfying conditions (a) and (b) of Corollary 3.4 and the hamiltonian coloring $h$ defined by (6)-(7) is an optimal hamiltonian coloring. For this we consider the following two cases for $B_{d}$.

Case-1: $d=2 k$. In this case, note that $B_{2 k}$ has $k(2 k-1)+1$ branches. Denote the branches of $B_{2 k}$ by $T_{i}, i=1,2, \ldots, k(2 k-1)+1$ such that $\left|T_{1}\right|>\left|T_{2}\right|=\ldots=\left|T_{k(2 k-1)+1}\right|$. Let $S=\left\{v: v \in V\left(T_{1}\right)\right\}$ and $S^{\prime}=\left\{v: v \in V\left(T_{i}\right), i=2,3, \ldots, k(2 k-1)+1\right\}$. Define an ordering $\left\{x_{0}, x_{1}, \ldots, x_{n}\right\}$ of $V\left(B_{2 k}\right)$ as follows: Let $x_{0}=w$ and for $1 \leq i \leq 4 k-4$, let

$$
x_{i}:= \begin{cases}v \in S, & \text { If } i \text { is odd, } \\ v \in S^{\prime}, & \text { If } i \text { is even. }\end{cases}
$$

For $4 k-3 \leq i \leq n-1$, let

$$
x_{i}:=v \in S^{\prime} .
$$

In above ordering $v \in X$, where $X=S, S^{\prime}$ we mean any $v$ from $X$ without repetition. Note that $\mathcal{L}\left(x_{0}\right)+\mathcal{L}\left(x_{n-1}\right)=1$ and $x_{i}, x_{i+1}(0 \leq i \leq n-1)$ are in different branches of $B_{2 k}$.

Case-2: $d=2 k+1$. In this case, note that $B_{2 k+1}$ has $k(2 k+1)+1$ branches. Denote the branches of $B_{2 k+1}$ by $T_{i}, i=1,2, \ldots, k(2 k+1)+1$ such that $\left|T_{1}\right|>\left|T_{2}\right|=\ldots=\left|T_{k(2 k+1)+1}\right|$. Let $S=\left\{v: v \in V\left(T_{1}\right)\right\}$ and $S^{\prime}=\left\{v: v \in V\left(T_{i}\right), i=2,3, \ldots, k(2 k+1)+1\right\}$. Define an ordering $\left\{x_{0}, x_{1}, \ldots, x_{n}\right\}$ of $V\left(B_{2 k+1}\right)$ as follows: Let $x_{0}=w$ and for $1 \leq i \leq 4 k$, let

$$
x_{i}:= \begin{cases}v \in S, & \text { If } i \text { is odd, } \\ v \in S^{\prime}, & \text { If } i \text { is even. }\end{cases}
$$


For $4 k+1 \leq i \leq n-1$, let

$$
x_{i}:=v \in S^{\prime} .
$$

Again in above ordering $v \in X$, where $X=S, S^{\prime}$ we mean any $v$ from $X$ without repetition. Note that $\mathcal{L}\left(x_{0}\right)+\mathcal{L}\left(x_{n-1}\right)=1$ and $x_{i}, x_{i+1}(0 \leq i \leq n-1)$ are in different branches of $B_{2 k+1}$ which completes the proof.

Example 4.5. An optimal hamiltonian coloring of $B_{3}, B_{5}$ and $B_{4}, B_{6}$ is shown in Fig. 2.

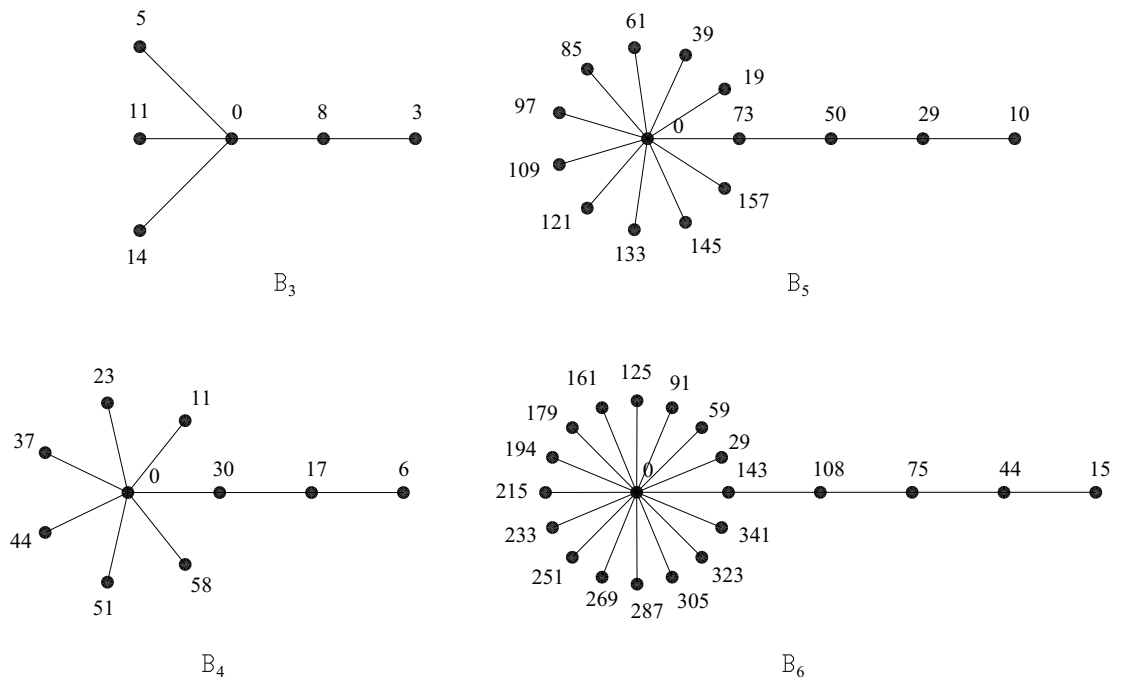

Figure 2: An optimal hamiltonian coloring of $B_{3}, B_{5}$ and $B_{4}, B_{6}$.

We claim that the lower bound given in Theorem 3.1 is better than the lower bound given in [3, Theorem 4] for broom trees $B_{d}(d=2 k, 2 k+1)$ except $B_{2}$. For broom tree $B_{2}$, both lower bounds are identical. Denote the right-hand side of $(3)$ and [3, Equation-(2)] by $l b_{W}(T)$ and $l b(T)$ respectively. That is, $l b_{W}(T)=(n-1)(n-1-\zeta(T))+\zeta^{\prime}(T)-2 \mathcal{L}_{W}(T)$ and $l b(T)=$ $(n-1)(n-1-\varepsilon(T))+\varepsilon^{\prime}(T)-2 \mathcal{L}(T)$. Since $h c\left(B_{d}\right)=l b_{W}\left(B_{d}\right)(d=2 k, 2 k+1 \geq 3)$, it is enough to prove that $l b_{W}\left(B_{d}\right)-l b\left(B_{d}\right)>0$ to justify our claim.

Theorem 4.6. Let $k \geq 1$ be any integer. Then $l b_{W}\left(B_{d}\right)-l b\left(B_{d}\right)>0$, where $d=2 k, 2 k+1 \geq 3$.

Proof. We consider the following two cases.

Case-1: $d=2 k$. In this case, $l b_{W}\left(B_{d}\right)-l b\left(B_{d}\right)=4 k(k-1)^{2}>0$.

Case-2: $d=2 k+1$. In this case, $l b_{W}\left(B_{d}\right)-l b\left(B_{d}\right)=4 k^{3}-2 k^{2}-k+1=k\left[(2 k-1)^{2}+2(k-\right.$ 1)] $+1>0$ which completes the proof.

\section{Concluding remarks}

In [4], Chartrand et al. proved that for $n \geq 1$,

$$
\operatorname{hc}\left(K_{1, n-1}\right)=(n-2)^{2} \text {. }
$$

This result can be proved using Corollary 3.4 as follows. The total detour level of $K_{1, n-1}$ is $\mathcal{L}\left(K_{1, n-1}\right)=n-1$ and $\left|W\left(K_{1, n-1}\right)\right|=1$. Substituting $\mathcal{L}\left(K_{1, n-1}\right)=n-1$ in (3), we obtain that the right-hand side of 16 is a lower bound for hc $\left(K_{1, n-1}\right)$ and it is easy to find a hamiltonian coloring whose span equal to this lower bound (refer [4]). 
A tree is said to be a caterpillar $C$ if it consists of a path $v_{1}, v_{2}, \ldots, v_{m}(m \geq 3)$, called the spine of $C$, with some hanging edges known as legs, which are incident to the inner vertices $v_{2}, v_{3}, \ldots, v_{m-1}$. If $d\left(v_{i}\right)=d$ for $i=2,3, \ldots, m-1$, then denote the caterpillar by $C(m, d)$. In [9], Shen et al. proved that for any positive integers $m \geq 3$ and $d \geq 3$,

$$
h c(C(m, d)):= \begin{cases}\frac{2 d-3}{2 d-2}(n-2)^{2}+\frac{d-1}{2}, & \text { If } m \text { is odd, } \\ \frac{2 d-3}{2 d-2}(n-2)^{2}, & \text { If } m \text { is even. }\end{cases}
$$

This result can also be proved using Corollary 3.4 as follows. The order $n$ and total detour level $\mathcal{L}(C(m, d))$ are given by

$$
\begin{gathered}
n:= \begin{cases}(2 k-1)(d-1)+2, & \text { If } m=2 k+1, \\
2 k(d-1)-2(d-2), & \text { If } m=2 k .\end{cases} \\
\mathcal{L}(C(m, d)):= \begin{cases}(k(k+1)-1)(d-1)+1, & \text { If } m=2 k+1, \\
k(k-1)(d-1), & \text { If } m=2 k .\end{cases}
\end{gathered}
$$

Substituting (18) and (19) into (3) we obtain right-hand side of (17) is a lower bound for $\mathrm{hc}(C(m, d))$ and it is easy to find a hamiltonian coloring whose span equal to this lower bound (refer [9]).

\section{References}

[1] R. Balakrishnan, K. Viswanathan Iyer, and K. Raghavendra: Wiener index of two special trees, MATCH Commun. Math. Comput. Chem., 57, 385-392 (2007).

[2] D. Bantva, S. Vaidya and S. Zhou: Radio number of trees, Discrete Applied Math., 217, 110-122 (2017).

[3] D. Bantva: On hamiltonian colorings of trees, In: S. Govindrajan and A. Maheshwari (eds.), Algorithms and Discrete Applied Math., CALDAM 2016, LNCS, 9602, 49-60, Springer, Heidelberg (2016).

[4] G. Chartrand, L. Nebeský, P. Zhang: Hamiltonian coloring of graphs, Discrete Applied Math., 146, 257-272 (2005).

[5] G. Chartrand, L. Nebeský, P. Zhang: On hamiltonian colorings of graphs, Discrete Math., 290, 133-143 (2005).

[6] G. Chartrand, P. Zhang: Distance in Graphs - Taking the Long View, AKCE J. Graphs. Combin., 1(1), 1-13 (2004).

[7] R. Khennoufa, O. Togni: A note on radio antipodal colourings of paths, Mathematica Bohemica, 130(3), 277-282 (2005).

[8] D. Liu: Radio number of trees, Discrete Math., 308, 1153-1164 (2008).

[9] Y. Shen, W. He, X. Li, D. He, X. Yang: On hamiltonian colorings for some graphs, Discrete Applied Math., 156, 3028-3034 (2008).

[10] D. West: Introduction to Graph Theory, Prentice-Hall of India, 2001. 